\title{
Technè
}

La science au service de l'histoire de l'art et de la préservation des biens culturels

42 | 2015

Science et conservation

\section{Régénération continue des bains de PEG utilisés pour la consolidation des bois archéologiques gorgés d'eau}

Continuous regeneration of PEG baths used to consolidate waterlogged archaeological wood

Loïc Caillat, Laure Meunier-Salinas et Marie-Amande Coignard

\section{OpenEdition}

\section{Journals}

Édition électronique

URL : http://journals.openedition.org/techne/7290

DOI : $10.4000 /$ techne.7290

ISSN : 2534-5168

Éditeur

C2RMF

Édition imprimée

Date de publication : 1 décembre 2015

Pagination : 115-120

ISBN : 978-2-7118-6249-8

ISSN : $1254-7867$

\section{Référence électronique}

Loïc Caillat, Laure Meunier-Salinas et Marie-Amande Coignard, «Régénération continue des bains de PEG utilisés pour la consolidation des bois archéologiques gorgés d'eau », Technè [En ligne], 42 | 2015, mis en ligne le 01 décembre 2015, consulté le 11 mars 2021. URL : http://journals.openedition.org/ techne/7290; DOI : https://doi.org/10.4000/techne.7290

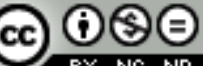

La revue Technè. La science au service de l'histoire de l'art et de la préservation des biens culturels est mise à disposition selon les termes de la Licence Creative Commons Attribution - Pas d'Utilisation Commerciale - Pas de Modification 4.0 International. 
Loïc Caillat

Laure Meunier-Salinas

Marie-Amande Coignard
Régénération continue des bains de PEG utilisés pour la consolidation des bois archéologiques gorgés d'eau

Continuous regeneration of PEG baths used to consolidate waterlogged archaeological wood
Résumé. ARC-Nucléart a élaboré un système de régénération des bains de polyéthylène glycol (PEG), utilisés dans le traitement de consolidation des bois archéologiques gorgés d'eau et progressivement pollués par différentes bactéries, par les substances dissoutes et les particules apportées par les bois eux-mêmes. La nouveauté de cette approche est le remplacement de l'usage des biocides par l'extraction et la neutralisation des contaminants et ce suivant quatre axes: le retrait des particules et des levures par micro-filtration, celui des ions par résines à lit fluidisé, la désinfection de la solution de PEG par passage devant une lampe UVc et l'aération des bains. Ces mesures permettent de régénérer les bains et donc de réduire sensiblement l'impact économique et environnemental des traitements tout en améliorant leur qualité.

Mots-clés. Bois archéologiques, recyclage des bains de traitement au PEG, filtration, UVc.
Abstract. ARC-Nucléart has developed a system for regenerating PEG (polyethylene glycol) baths, used in the treatment for consolidating waterlogged archaeological wood progressively degraded by bacteria, by substances that have dissolved in the water and by particles added by the wood itself. The novelty of this approach lies in its method of replacing the use of biocides with a fourfold method of extraction and neutralizing contaminants: removal of particules and yeasts by micro-filtration; removal of ions by fluidized beds of resin/activated charcoal; disinfection of the PEG solution by running it past an UVC lamp and by air bullage (oxygenation) of baths. Using these measures to regenerate the baths markedly reduces the economic and environmental impact of the treatments at the same time as improving their quality.

Keywords. Archaeological wood, recycling PEG treatment baths, filtration, UVC.

\section{Introduction}

Les bois archéologiques "gorgés d'eau » sont ainsi appelés parce que l'eau est à la fois leur milieu de conservation et l'élément qui va, au fil du temps, lentement solubiliser la cellulose du bois et occuper l'espace ainsi libéré par la perte de matière. L'eau permet alors de préserver la forme de l'objet en évitant l'effondrement du matériau. Le traitement par immersion dans des solutions d'eau et de polyéthylène glycol (PEG) constitue la méthode la plus couramment utilisée dans le monde par les ateliers de conservation spécialisés.

Les PEG sont des molécules à haut poids moléculaire issues de l'industrie de la pétrochimie. Ils sont constitués d'une longue chaîne carbonée aliphatique, plus ou moins hydrosoluble grâce à ses fonctions éthers et alcools.

Cette famille de molécules présente l'avantage de ne pas être toxique, qualité qui se transforme en inconvénient puisqu'un certain nombre de micro-organismes peuvent coloniser ce milieu. Ils peuvent dès lors constituer un risque sur le plan sanitaire, mais également pour la conservation des objets. Ces micro-organismes, bactéries aérobies, anaérobies et levures pour ne citer que les plus importants, proviennent essentiellement du sédiment d'enfouissement et sont véhiculés par l'eau présente dans les bois archéologiques.

Pour faire face à ce problème récurrent de contamination biologique, l'atelier ARC-Nucléart a progressivement développé une stratégie fondée sur la filtration et la désinfection en continu afin de recycler en permanence les 130000 litres de solution de PEG qu'il utilise. Ceci a permis dans le même temps de maintenir la qualité des bains durant tout le processus d'imprégnation et de diminuer les rejets et les achats de matière première. Ainsi, il a été possible de réduire significativement l'impact de ces traitements sur l'environnement.

\section{La problématique des solutions de traitement au PEG}

Les imprégnations de consolidation des bois durent au minimum huit mois, soit généralement deux bains de quatre mois à concentration croissante, $20 \%$ puis $35 \%$ massique de PEG. La faible concentration du premier bain permet de limiter la pression osmotique et d'éviter que l'eau, très mobile, ne 
sorte de l'objet avant que le consolidant visqueux (PEG) ait pu y pénétrer.

Au départ incolore et translucide, le bain de polyéthylène glycol se charge progressivement en matières en suspension (MES) au cours du traitement. Ces particules peuvent être de nature minérale (restes de sédiment), organique mais inerte (particules de bois), ou encore constituées de microorganismes en activité, des bactéries ou des levures qui prolifèrent dans le milieu liquide (organismes planctoniques). La solution de PEG se charge, dans le même temps, en substances dissoutes telles que des colorants (tanins issus du bois, sels contenant du fer issus des parties métalliques corrodées). On trouve également d'autres substances dont éventuellement des produits biocides et les sous-produits de leur dégradation.

Il en résulte un trouble du liquide, dû à la quantité de MES et, lorsque celle-ci devient trop importante, une opacification totale du bain, accompagnée d'odeurs désagréables (fig. 1). En dehors de l'aspect purement sanitaire et de l'image négative que peut renvoyer un tel bain, des interactions avec les objets sont possibles, potentiellement néfastes à leur conservation : recouvrement des surfaces par un biofilm, coloration noire et accumulations de produits instables à l'air ou de composés organiques volatils (COV) indésirables.

Une des altérations classiques des objets archéologiques en cours de traitement est l'apparition d'un biofilm. Les bactéries anaérobies sulfato-réductrices, sensibles à l'oxygène, se mettent alors à proliférer sous le biofilm et, par leur activité métabolique en présence de sulfates, conduisent à la formation de soufre à l'état réduit qui va se combiner avec le fer, présent sous forme d'oxyde, en composés ferro-soufrés, les sulfures de fer qui vont donner une coloration noire au bois. En outre, le film bactérien qui enveloppe les objets peut limiter les échanges eau-PEG et freiner l'imprégnation de consolidant.

En raison de l'accumulation des polluants - particules, micro-organismes, colorants, biocides, gaz dissous ou colloïdes non-décantables -, les bains de PEG sont alors impropres à une réutilisation à l'issue du traitement.

L'achat du PEG et son élimination par incinération représentent une part très significative du coût global d'un

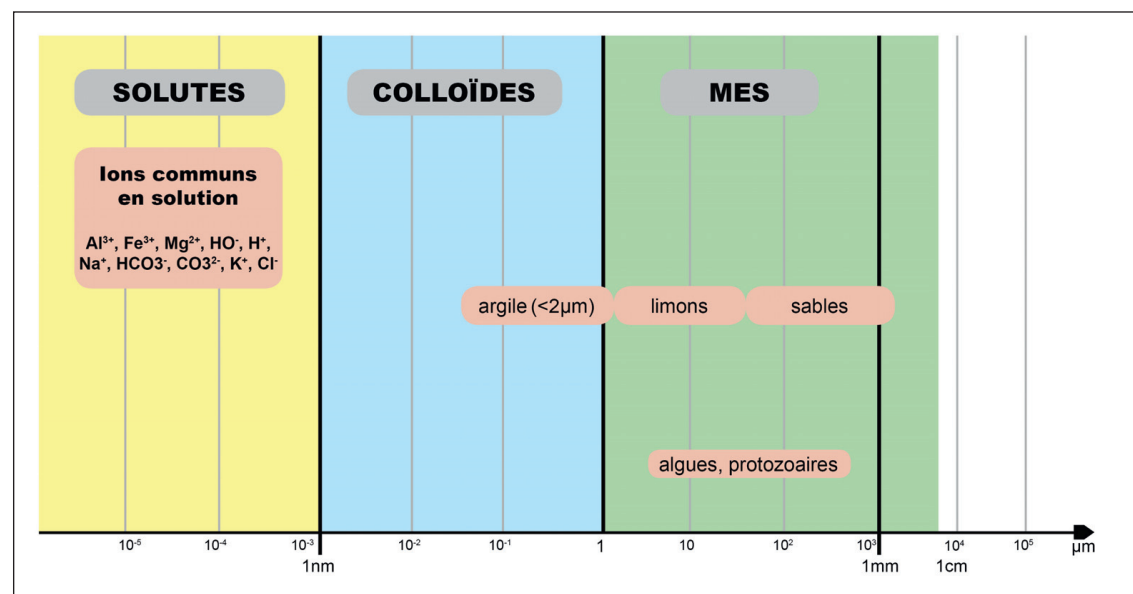

Fig. 1. Les matières en suspension (MES) à l'origine de la pollution des bains de PEG. traitement. La mise aux déchets d'importantes quantités de solutions de PEG est donc un non-sens économique et environnemental, alors que le PEG lui-même n'est pas altéré durant le traitement et qu'il garde toutes ses propriétés de consolidant.

Le premier réflexe pour allonger la durée de vie des bains est d'ajouter des biocides afin de limiter la prolifération des micro-organismes et l'accumulation de produits indésirables. Les biocides à spectre large sont particulièrement efficaces, mais ils sont toxiques, faiblement biodégradables et s'accumulent dans les bains. De plus, les normes actuelles sur les produits chimiques (norme Reach ${ }^{1}$ ) limitent la gamme autorisée et interdisent déjà les plus toxiques, qui sont aussi les plus efficaces. Pour avoir une action sur l'ensemble des microorganismes, il faudrait donc effectuer des combinaisons complexes, difficiles à mettre en ouvre, ce qui pose le problème des interactions non maîtrisées entre plusieurs principes actifs.

\section{Réflexion sur les améliorations possibles}

Une réflexion a été menée pour parvenir à maintenir constante la qualité des bains, d'abord en sélectionnant d'autres biocides puis en choisissant de s'en affranchir. Le principe était donc, non pas d'ajouter un ou des produits actifs, mais au contraire d'extraire le plus possible les éléments qui posent problème. Comment régénérer les bains afin de retirer ce qui les rend impropres à la réutilisation ? Peut-on transformer cette phase passive de traitement en une phase active?

La solution mise en place comprend un ensemble d'actions qui ont pour but de transformer des bains stationnaires passifs et non contrôlés en bains dynamiques et méticuleusement surveillés, avec un certain nombre de paramètres indicateurs de leur bonne qualité.

Nous avons choisi d'adapter à nos équipements des techniques utilisées dans le domaine de la dépollution de l'eau. Les méthodes décrites ci-dessous - une aération active des bains, la microfiltration et un traitement UVc germicide - sont combinées en un système global (fig. 2) qui vise à obtenir le bain le plus sain possible, contenant quasi-exclusivement du PEG et de l'eau.

\section{Oxygénation des bains}

Le système d'aération des bains («skimmer " de piscine ou autres dispositifs immergés de bullage) est facile à mettre en ouvre pour empêcher la prolifération de la flore anaérobie qui représente une partie de la population bactérienne à l'origine de troubles, de mauvaises odeurs, de colorations noires des objets, sans parler des risques sanitaires 


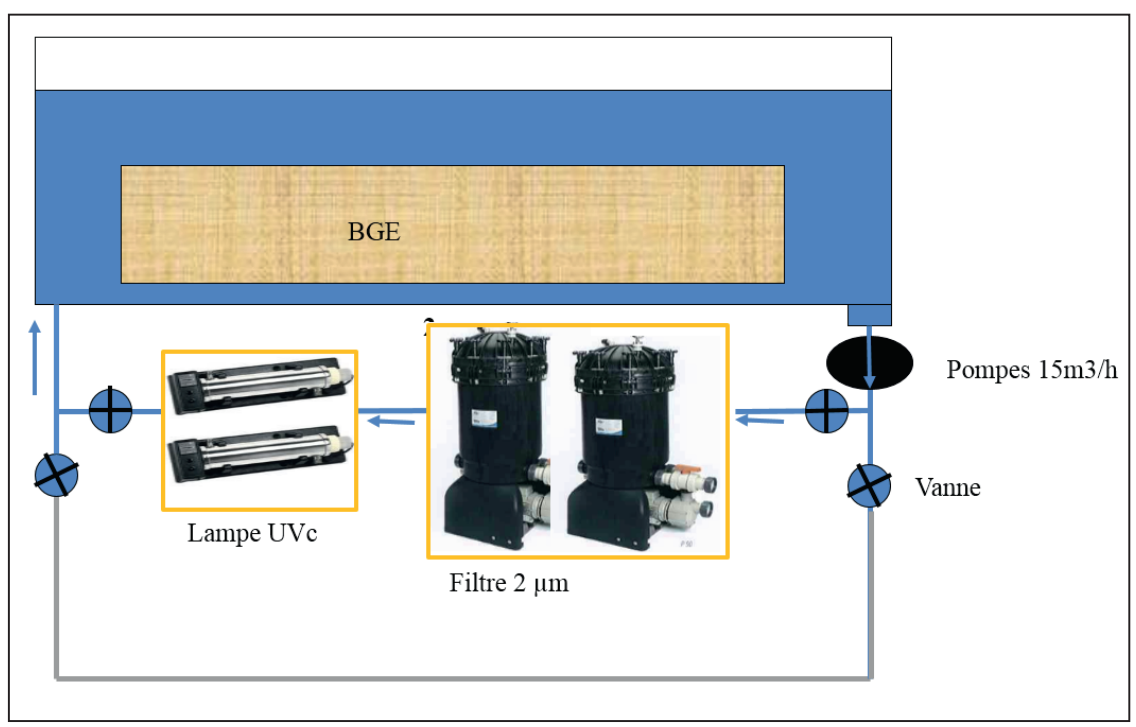

Fig. 2. Schéma du système global de nettoyage : circuit, filtres, porte-filtres et lampes. encombrement réduits, de fixer une quantité plus grande de particules, de retarder le colmatage et de minimiser les interventions de maintenance. La durée de vie de ces filtres peut être longue puisque, lorsqu'ils sont colmatés, ils peuvent être nettoyés au jet d'eau un certain nombre de fois et réutilisés pendant plusieurs années. L'eau de nettoyage ne contient alors qu'un infime pourcentage de PEG, biodégradable à faible concentration, et des matières issues du milieu d'enfouissement.

Néanmoins, malgré leur efficacité, ces filtres ne suffisent pas à éliminer complètement le trouble du bain imputable à d'autres éléments, comme les particules colloïdales et les bactéries, de taille submicronique (de $0,4 \mu \mathrm{m}$ à quelques microns pour les bactéries).

Il existe un type de filtre à $0,2 \mu \mathrm{m}$ capable de retenir des éléments aussi petits, mais son utilisation a un coût très élevé car il est non lavable et à usage unique. La nano-filtration est donc réservée très ponctuellement à la capture des particules colloïdales pour lesquelles les filtres de $2 \mu \mathrm{m}$ sont inefficaces.

\section{Décontamination des bains par UVc}

Pour empêcher la colonisation de la solution de traitement par des bactéries aérobies favorisées par l'aération, le bain est exposé à un rayonnement UVc. L'irradiation UVc constitue une méthode physique très employée dans la potabilisation de l'eau. Pour que les UVc soient efficaces malgré leur faible pouvoir de pénétration dans l'eau, le liquide circule en très faible épaisseur autour de la source. Les UVc agissent sur les micro-organismes en altérant leur $\mathrm{ADN}$, ce qui bloque leur mécanisme de réplication. Le processus de multiplication qui permet aux populations microbiennes de croître de manière exponentielle et d'envahir l'ensemble du milieu est ainsi arrêté. La population microbiologique est maîtrisée et non pas éliminée. Le bain filtré est dirigé vers le dispositif UVc, constitué d'un tube inox à l'intérieur duquel est introduit un second tube en quartz de $50 \mathrm{~cm}$ de long pour environ $4 \mathrm{~cm}$ de diamètre. Dans l'espace entre les deux tubes - n'excédant pas $2 \mathrm{~cm}$-, le liquide est exposé à une irradiation UVc produite par un néon à vapeur de mercure d'une puissance de 36 watts. La durée de l'exposition est très courte, de l'ordre de la dizaine de secondes. Il s'agit d'une stérilisation flash qui permet de ne pas endommager le PEG, par ailleurs protégé de la photo-oxydation par l'eau qui l'entoure.

Pour obtenir de bons résultats avec la désinfection par UVc, le milieu doit être le plus transparent possible, donc débarrassé au maximum des MES par les filtres à particules déjà cités. Ce n'est pas toujours suffisant, le bain doit contenir
Fig. 3. Le matériel utilisé pour la filtration des bains, filtres à particules d'une maille de $2 \mu \mathrm{m}$; à droite, un filtre utilisé après lavage à l'eau en partie basse. @ A ARC-Nucléart. 
peu de substances dissoutes, en particulier peu de colorants (tanin, oxyde de fer) qui diminuent l'action des UVc.

\section{Filtration chimique}

Pour décolorer efficacement et éliminer les substances dissoutes, telles les molécules non chargées et les ions, il est utilisé un système de filtration qui consiste à retenir chimiquement, et non pas mécaniquement, les polluants. La filtration chimique est fondée sur un système en lit fluidisé, c'est-à-dire une technique de percolation consistant à faire passer le liquide à travers une colonne de matériau granulaire ayant une affinité chimique avec la substance à retirer. Dans notre cas, le lit fluidisé est contenu dans un cylindre creux de même format que les filtres à particules. Il est muni en son centre d'une bobine filtrante pour empêcher les grains du lit fluidisé de s'échapper vers le bain. Cette technique permet de retenir un grand nombre de substances, même si elles sont très diluées. Deux types de matériaux sont utilisés : le charbon actif, dont les grains très poreux permettent de retenir à la fois chimiquement et physiquement les grosses molécules et des gaz dissous ; les résines échangeuses d'ions, qui ont une affinité pour des molécules de plus petite taille à charge électrostatique positive ou négative. Les lits de résine et de charbon sont régulièrement remplacés (fig. 4). Ils constituent alors des déchets peu importants en volume, faciles à incinérer.

\section{Protocoles de traitement et de suivi des bains de PEG}

Grâce à son expérience dans la gestion des bains de PEG, ARC-Nucléart a établi des protocoles de traitement de plus en plus efficaces qui permettent d'associer les quatre dispositifs décrits précédemment : la micro-filtration, l'oxygénation par bullage, la stérilisation par UVc et la filtration chimique. Nous avons fait le choix de matériel standardisé afin de disposer de pièces interchangeables et de construire un système global, polyvalent et adaptable.

Pour parfaire le système, l'ajout ponctuel d'acide citrique, biodégradable et non toxique, peut être nécessaire pour éviter l'alcalinisation du bain (due en grande partie à la désionisation des solutions), afin de maintenir le bain à un $\mathrm{pH}$ compris entre 5 et 6 . Ce milieu légèrement acide est défavorable à la multiplication de la plupart des populations bactériennes. Cette adjonction, la seule que nous nous permettons encore, est essentielle pour limiter la formation de biofilm à l'interface bois-bain intervenant préférentiellement au-dessus de pH 7.

Pour connaître l'état microbiologique des bains, des analyses sont réalisées mensuellement à partir de prélèvements. Cela permet de vérifier le bon fonctionnement du système de régénération des solutions de PEG et d'intervenir de façon préventive sur le système avant que la qualité du bain ne se dégrade en cas de dysfonctionnement (encrassement du quartz du dispositif UVc, lampe UV grillée ou coloration trop importante du bain). D’autres paramètres, comme la température, le $\mathrm{pH}$, la concentration sont également suivis. Des
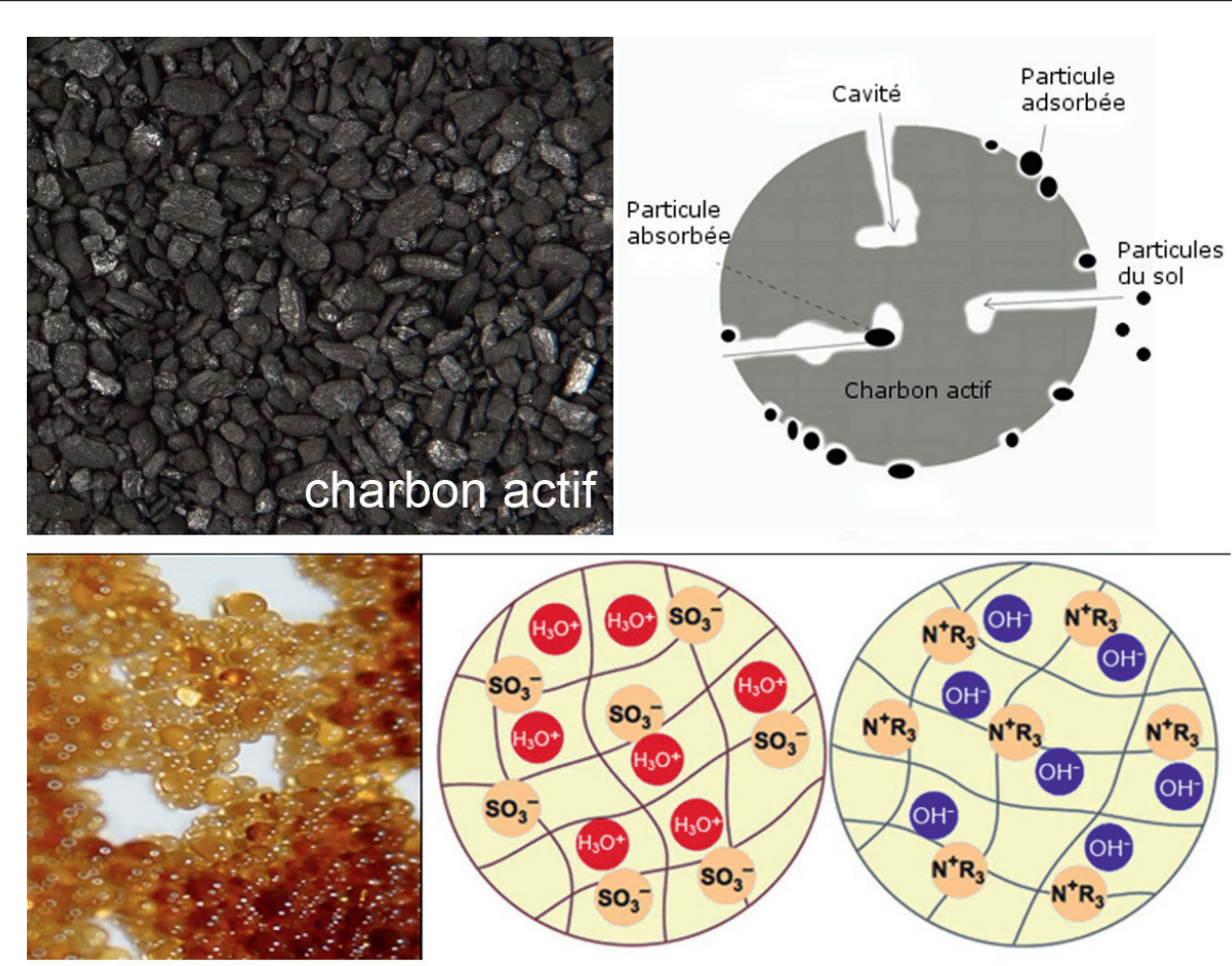

Fig. 4. Les filtres chimiques à lit fluidisé. Dans la partie haute, le charbon actif, dans la partie basse, la résine échangeuse d'ions. (c) ARC-Nucléart. 


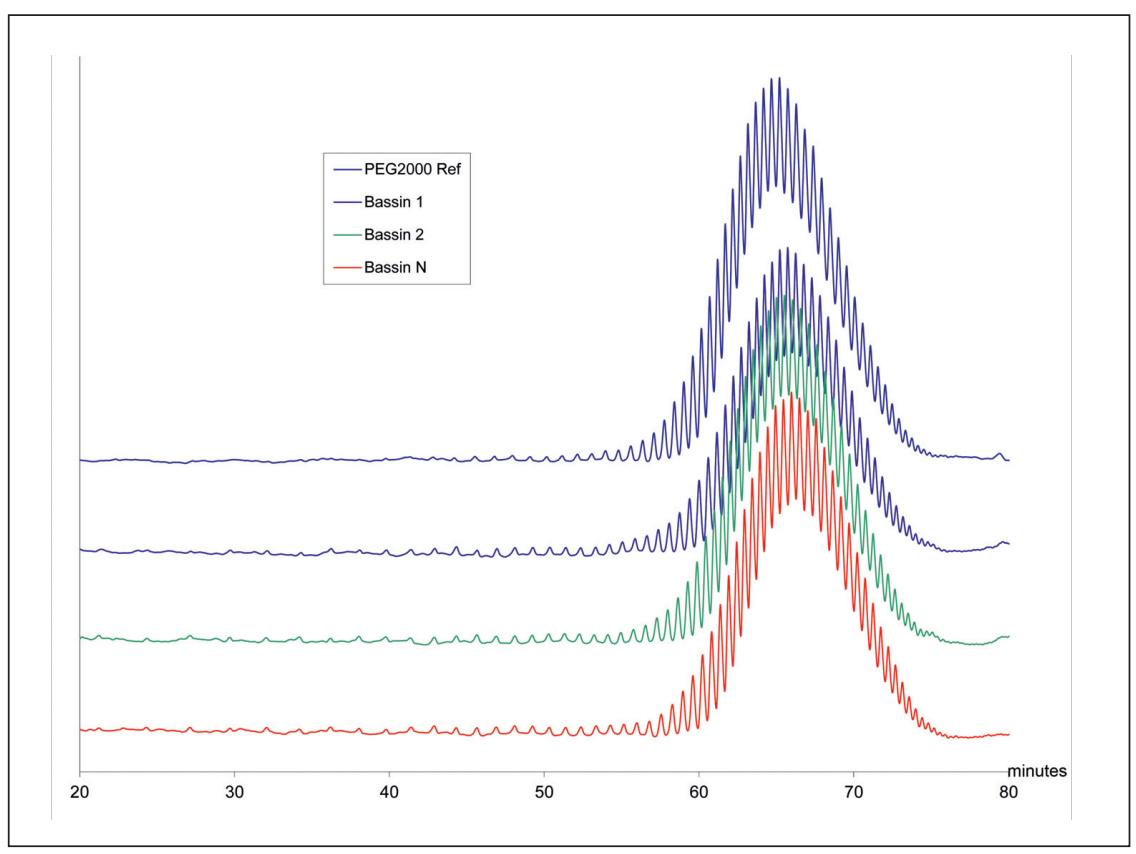

Fig. 5. Contrôle de la qualité du PEG exposé au rayonnement UVc par recherche de coupures de chaînes ; comparaison du temps d'élution sur colonne HPLC avec détection à $190 \mathrm{~nm}$ par rapport à un PEG neuf (courbe bleue du haut).

analyses sont effectuées pour déterminer la qualité du PEG. Pour cela, la longueur des chaînes moléculaires est contrôlée tous les six mois au moyen d'une analyse par chromatographie liquide à haute pression (HPLC) (fig. 5). Nous évaluons par ailleurs mensuellement, au moyen d'analyses par IRTF, l'oxydation du polymère, altération possible en raison de l'exposition continue au rayonnement UVc.

Ce suivi est réalisé par ARC-Nucléart depuis plus de cinq ans. L'absence d'évolution par rapport à des échantillons de PEG neuf indique que le consolidant n'est pas altéré par le recyclage des bains.

\section{Conclusion et perspectives}

La régénération des bains de PEG permet la réduction à la fois des coûts de fonctionnement et de l'impact sur l'environnement. Par ailleurs, la disparition des biocides entraîne une réduction de l'impact sur les opérateurs et sur la conservation à long terme des objets. La transparence des bains rend possible désormais une surveillance tout au long du traitement et chaque objet bénéficie d'un bain continûment propre, dans lequel il est le seul agent contaminant.

Ce qui était à la base une recherche d'économie et de durabilité est devenu un élément incontournable du traitement. Le résultat en termes de qualité est si probant qu'il apparaît désormais difficile de s'en passer dans les traitements au PEG (fig. 6). Pour ces raisons, des relations ont été établies avec d'autres ateliers souhaitant s'équiper en dispositifs de filtration continue.

Le risque d'un envahissement du bain par des micro-organismes et de la formation d'un biofilm sur les objets est toujours à envisager en cas de panne du système. Mais ce qui était un état de fait devient un accident ponctuel et gérable. Le bain, même fortement pollué, peut toujours être régénéré par filtration et UVc ; ce n'est toutefois pas le cas des surfaces (artefacts, étagères...) qui doivent être rincées à l'eau en fin de traitement si du biofilm s'est développé.

Un autre avantage, dû une fois de plus au phénomène d'osmose, demande encore à être quantifié. Du fait du mouvement naturel des ions depuis un milieu hypertonique (chargé) dans les bois vers un milieu hypotonique (dilué) dans le bain, l'assainissement progressif et constant du bain peut avoir un impact positif sur les objets eux-mêmes. Par conséquent, cette méthode de purification des bains, par l'appauvrissement constant du milieu en sels, peut être considérée comme une forme de nettoyage en volume des objets. Certes, ce processus est particulièrement lent, mais également continu, homogène, dynamique et, du fait du temps d'immersion, potentiellement efficace. Une étude devrait prochainement être initiée pour quantifier l'extraction des contaminants présents dans les bois, en particulier celle des composés soufrés et ferreux.

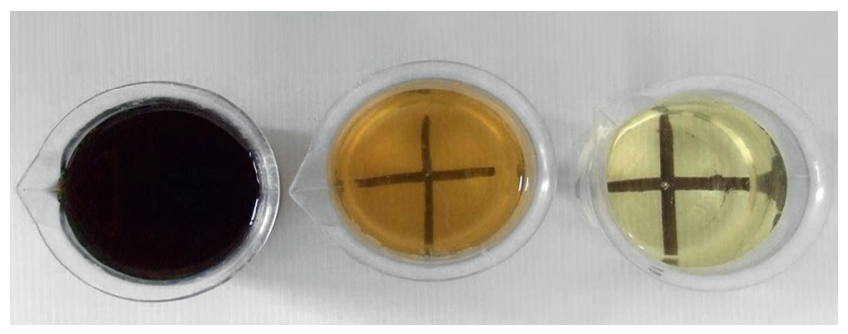

Fig. 6. La régénération d'un bain pollué par l'accumulation de particules et de substances dissoutes. À gauche, bain non traité ; au centre, bain traité par micro-filtration ; à droite, bain traité par micro-filtration et charbon actif. (c) ARC-Nucléart. 


\section{Notes}

1. Reach est un règlement européen $\left(n^{\circ} 1907 / 2006\right)$ entré en vigueur en 2007 pour sécuriser la fabrication et l'utilisation des substances chimiques dans l'industrie européenne. Il s'agit de recenser, d'évaluer et de contrôler les substances chimiques fabriquées, importées, mises sur le marché européen. D’ici 2018, plus de 30000 substances chimiques seront connues et leurs risques potentiels établis. L'Europe disposera ainsi des moyens juridiques et techniques pour garantir à tous un haut niveau de protection contre les risques liés aux substances chimiques.

\section{Bibliographie}

Björdal, C. G., \& Nilsson, T., 2001, "Observations on microbial growth during conservation treatment of waterlogged archaeological wood", Studies in conservation, 46 (3), p. 211220.

Cohen O., 1999, "The Kinneret Boat, Conservation and Exhibition", Proceedings of the $7^{\text {th }}$ ICOM group on Wet Organic Archaeological Materials Conference, Grenoble 1998, Published by ARC-Nucléart.
Daniel S. L., Rolston J. M., 2010, "Bacteria/ fungi: a growing concern for waterlogged wood", Wood conservation strategies, methods, material and case stories, WOAM, Greenville 26 May 2010.

Degrigny C., Baron G., Christodoulou P., Tran Q. K., Hiron X., 2002,

"Conservation of a collection of waterlogged composite rifles dating from the $17^{\text {th }}$ century recovered from the Brescou marine site", Proceedings of the $8^{\text {th }}$ ICOM group on Wet organic Archaeological Materials conference, Stockholm 2001, Published by ICOMCC, WOAM Group, Bremerhaven.

Dwards K. J., Gihring T. M., Banfield J.F., 1999, "Seasonal variations in microbial populations and environmental conditions in an extreme acid mine drainage environment", Applied and Environmental Microbiology, Rapport BRGM, August 1999, p. 3627-3632.

Haaken D., Dittmar T., Schmalz V., Worch E., 2014, "Disinfection of biologically treated wastewater and prevention of biofouling by UV/electrolysis hybrid technology: Influence factors and limits for domestic wastewater reuse", Water research, Elsevier, n ${ }^{\circ} 52$, p. 20-28.

Hassen A., Mahrouk M., Ouzari H., Cherif M., Boudabous A., Damelincourt J.-J., 2000, "UV disinfection of treated wastewater in a large scale pilot plant and inactivation of selected bacteria in a laboratory UV device", Bioresource Technology, vol. 74, p. 141-150.

Memet J.-B. and Tran K., 2005, "Development of a conservation treatment process adapted to archaeological iron/waterlogged wood composites", Proceedings of the $9^{\text {th }}$ ICOM group on Wet organic Archaeological Materials conference, Copenhagen 2004, Published by ICOMCC, WOAM Group, Bremerhaven.

Preston J., Smith A. D., Schofield E. J., Chadwick A. V., Jones M. A., Watts, J. E. M., 2014, "The Effects of Mary Rose Conservation Treatment on Iron Oxidation Processes and Microbial Communities Contributing to Acid Production in Marine Archaeological Timbers", publication en ligne, Plosone.

Rakotonirainya M. S., Caillat L., Héraud C., Memet J.-B., Tran Q. K., 2007, "Effective biocide to prevent microbiological contamination during PEG impregnation of wet archaeological iron-wood artefacts", Journal of

Cultural Heritage, Volume 8, Issue 2, April-June 2007, p. 160-169. 